\title{
Growth kinetics and morphological analysis of homoepitaxial GaAs fins by theory and experiment
}

\author{
Marco Albani, ${ }^{1}$ Lea Ghisalberti, ${ }^{2}$ Roberto Bergamaschini, ${ }^{1,}{ }^{*}$ Martin Friedl, ${ }^{2,} \dagger$ Marco Salvalaglio, ${ }^{3}$ Axel \\ Voigt, ${ }^{3,4}$ Francesco Montalenti, ${ }^{1}$ Gözde Tütüncüoglu, ${ }^{2}+{ }^{\ddagger}$ Anna Fontcuberta i Morral, ${ }^{2}$ and Leo Miglio ${ }^{1}$ \\ ${ }^{1}$ L-NESS and Dept. of Materials Science, Università di Milano-Bicocca, 20125, Milano, Italy \\ ${ }^{2}$ Laboratory of Semiconductor Materials, École Polytechnique Fédérale de Lausanne, EPFL, 1015, Lausanne, Switzerland \\ ${ }^{3}$ Institute of Scientific Computing, Technische Universität Dresden, 01062, Dresden, Germany \\ ${ }^{4}$ Dresden Center for Computational Materials Science (DCMS), \\ Technische Universität Dresden, 01062 Dresden, Germany
}

(Dated: August 27, 2018)

\begin{abstract}
Nanoscale membranes have emerged as a new class of vertical nanostructures that enable the integration of horizontal networks of III-V nanowires on a chip. In order to generalize this method to the whole family of III-Vs, progress in the understanding of the membrane formation by selective area epitaxy in oxide slits is needed, in particular for different slit orientations. Here, it is demonstrated that the shape is primarily driven by the growth kinetics rather than determined by surface energy minimization as commonly occurs for faceted nanostructures. To this end, a phase-field model simulating the shape evolution during growth is devised, in agreement with the experimental findings for any slit orientations, even when the vertical membranes turn into multi-faceted fins. This makes possible to reverse-engineer the facet-dependent incorporation times, which were so far unknown, even for common low-index facets. The compelling reproduction of the experimental morphologies demonstrates the reliability of the growth model and offers a general method to determine microscopic kinetic parameters governing out-of-equilibrium three-dimensional growth.
\end{abstract}

PACS numbers: 81.10.Aj, 81.07.-b, 81.05.Ea, 68.65.-k

\section{INTRODUCTION}

Selective Area Epitaxy (SAE) is a widely used technique to drive the growth of nanostructures that are not achievable by direct-deposition onto a bare substrate. Confinement of the growth within openings defined in the mask by top-down patterning imposes particular constraints on the growth and development of the resulting structures, possibly leading to interesting morphologies. GaAs nanowires have been successfully obtained on (111)B GaAs and Si substrates by Metal-Organic Chemical Vapor Deposition (MOCVD) ${ }^{1-3}$, by opening apertures in the oxide layer with widths on the order of $100 \mathrm{~nm}$. By extending the slits in the $<11-2\rangle$ direction up to several microns, vertical nanomembranes (NMs) are obtained, as reported in the literature for both $\mathrm{MOCVD}^{4,5}$ and Molecular Beam Epitaxy $(\mathrm{MBE})^{6}$. These NMs have shown to exhibit exceptional optical properties, as a consequence of their perfect crystal structure and absence of twinning defects ${ }^{7}$, a feature that prevents also the occurrence of twinning-driven tilting, as in the case of nanowires ${ }^{8}$. In addition, it was recently demonstrated that these NMs can act as templates for the growth of horizontally aligned InAs nanowires ${ }^{9}$. The approach is wafer-scalable, opening the path towards integration of III-V nanowire networks on a chip, and providing a platform for the realization of advanced concepts in next generation computing schemes ${ }^{10}$.

A scanning electron microscope (SEM) image of a typical array of GaAs NMs is reported in Fig. 1. The majority of the NM is defined by $\{110\}$ facets with a (111)B faceted top. Small $\{113\}$ facets are recognizable at the sides of the top facet. Based on first principle surface energy calculations ${ }^{11}$, the absence of $\{111\} \mathrm{A}$ facets looks reasonable for the As-rich growth conditions exploited here $(\mathrm{V} / \mathrm{III}$ ratio $\sim 10)$, while the absence of $\{100\}$ facets contrasts with the expectation from the equilibrium Wulff shape of GaAs, as their surface energy should be still favorable. Moreover, the prominent tendency toward vertical growth, yet preserving the narrow crosssection initially enforced by the oxide slit, disagrees with the thermodynamic criterion of surface energy minimization. As we show here, indeed, the growth of the NMs is primarily driven by kinetics. In contrast to equilibrium conditions, the kinetic growth regime is not yet fully understood, as almost nothing is known about the different incorporation dynamics on the crystal facets, even for common low-index facets. Still, these differences greatly affect the evolution of the growing crystals, resulting in facet growth velocities that may vary even by a couple of orders of magnitude.

Modeling facet-dependent growth kinetics by exploiting atomistic approaches is extremely challenging, not only due to the lack of microscopic-scale parameters, but also because of severe limitations in both spatial and temporal scales. We have, therefore, devised a reverseengineering strategy to extract quantitative information about key parameters, such as the incorporation times for the different crystal facets. First, we considered new experiments by performing the growth on circular arrays, as obtained by rotating the rectangular growth slit around a given center on the (111)B substrate, similarly to the work of Ref. 12. Second, we applied a state-of-the-art continuum growth model, incorporating the main kinetic 

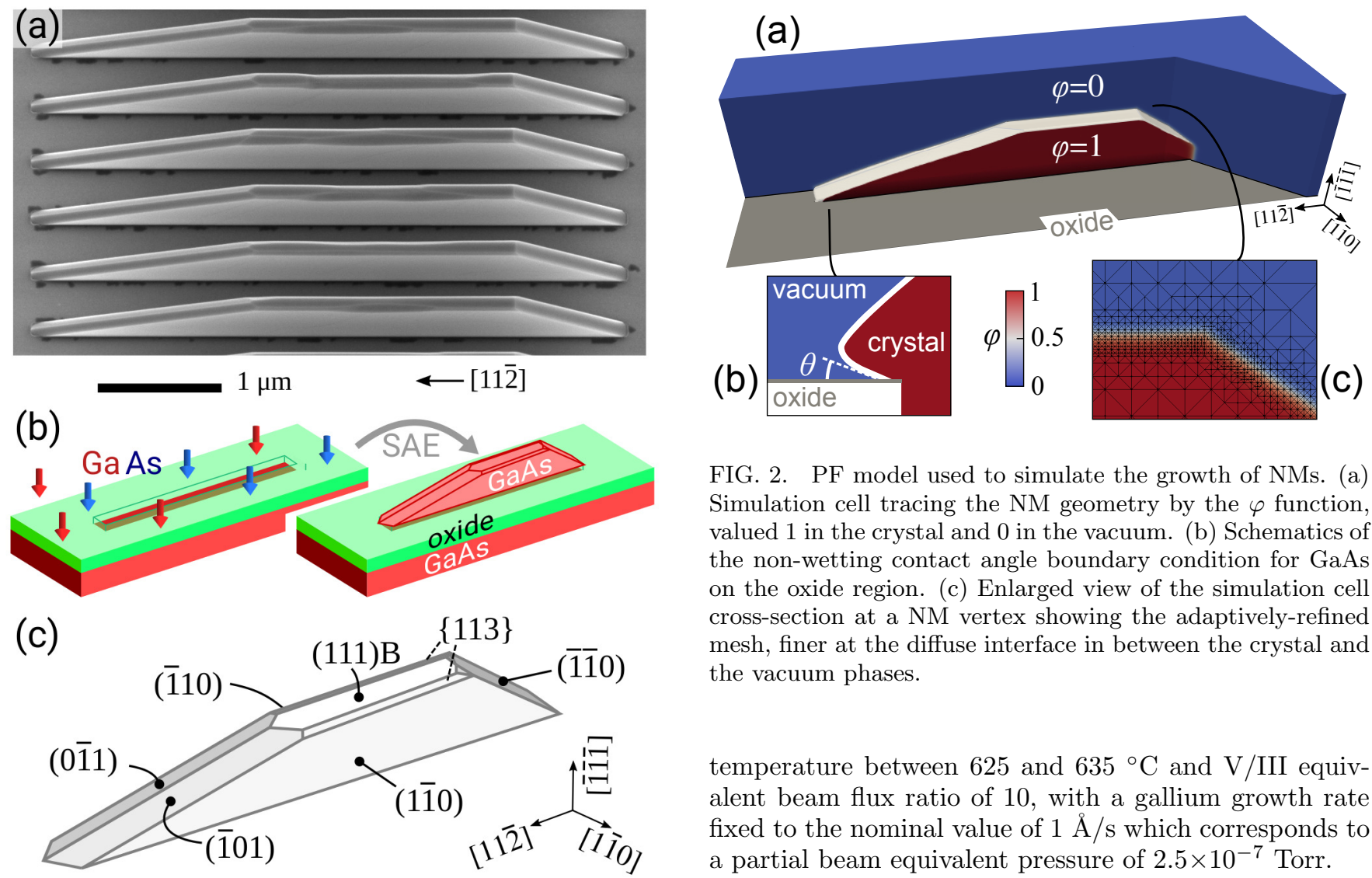

FIG. 2. PF model used to simulate the growth of NMs. (a) Simulation cell tracing the NM geometry by the $\varphi$ function, valued 1 in the crystal and 0 in the vacuum. (b) Schematics of the non-wetting contact angle boundary condition for GaAs on the oxide region. (c) Enlarged view of the simulation cell cross-section at a NM vertex showing the adaptively-refined mesh, finer at the diffuse interface in between the crystal and the vacuum phases.

temperature between 625 and $635{ }^{\circ} \mathrm{C}$ and V/III equivalent beam flux ratio of 10 , with a gallium growth rate fixed to the nominal value of $1 \AA / \mathrm{s}$ which corresponds to a partial beam equivalent pressure of $2.5 \times 10^{-7}$ Torr.

\section{PHASE-FIELD MODEL}

view of a typical array of NMs grown by Molecular Beam Epitaxy on (111)B GaAs substrate (b) Schematics of the Selective Area Epitaxy procedure. (c) Schematic drawing of a NM shape including the main facets observed experimentally.

contributions, so that the experimentally-observed morphological changes of the NMs as a function of slit orientation could be compared with simulation results.

\section{EXPERIMENTS}

The GaAs nanoscale membranes are grown by MBE. The substrate is prepared with a standard procedure for $\mathrm{SAE}$ as in Ref. 6. First, a layer of $30 \mathrm{~nm} \mathrm{SiO}_{2}$ is deposited onto a 2-inch (111)B GaAs substrate by PlasmaEnhanced Chemical Vapor deposition (PECVD). The resist ZEP 5250, is then spin-coated on the $\mathrm{SiO}_{2}$ layer which in turn is exposed to the electron beam lithography process to define the desired pattern. The apertures in the oxide are obtained by dry-etching with a mixture of $\mathrm{CH}_{3} \mathrm{~F} / \mathrm{SF}_{6}$. The final removal of the resist is carried out by 10 min of oxygen-plasma, followed by acetone and IPA cleaning. Before being grown, each sample is annealed and degassed at $350^{\circ} \mathrm{C}$ in ultra-high vacuum conditions. The growth conditions of the nanoscale membranes have been optimized, resulting with the following parameters:

The continuum model developed by J.W. Cahn and J.E. Taylor in Ref. 13 is exploited to study the growth of the GaAs NMs. This is numerically implemented within a phase-field approach $(\mathrm{PF})^{14,15}$, which is very effective in simulating complex, three-dimensional structures. The crystal morphology is traced implicitly by means of an order parameter, i.e. the $\mathrm{PF}$ function $\varphi$, with value 1 into the crystal and 0 in the surrounding vacuum region, as shown in Fig. 2(a). The surface profile is then identified as the $\varphi=0.5$ isoline in the diffuse-interface region (of width $\varepsilon$ ) where $\varphi$ goes smoothly from 1 to 0 . The whole substrate region out of the growth slit, corresponding to the bottom boundary of the simulation cell, is assumed to be covered by oxide as for SAE, thus resulting in a nonwetting contact condition for the GaAs crystal, according to Young's law (see Fig. 2(b)).

Even if the GaAs growth necessitates both Ga and As to proceed, here we explicitly consider just the dynamics of $\mathrm{Ga}$ adatoms on the growing surface. Although As is expected to play a role in the growth dynamics ${ }^{16}$, supply of it from the gaseous phase is not a limiting factor in case of high V/III ratio (as for the present experiments) and its reaction kinetics with $\mathrm{Ga}$ is implicitly considered as contributing to the incorporation rate of $\mathrm{Ga}$ into GaAs. The profile evolution is traced by the variation in time of the $\varphi$ field itself, as due to both material deposition and 
redistribution by surface diffusion:

$$
\frac{\partial \varphi}{\partial t}=F(\hat{\mathbf{n}})|\nabla \varphi|+\nabla \cdot[M(\varphi) \nabla \varphi]
$$

with $\hat{\mathbf{n}}=-\nabla \varphi /|\nabla \varphi|$ the local surface normal. The first addendum accounts for deposition with a rate $F(\hat{\mathbf{n}})$, mimicking the Ga flux in an MBE reactor (see Ref. 17 and Supplementary Material). Flux shielding effects on the NM sidewalls by neighboring structures ${ }^{18}$ are not considered. We also neglect Ga supply from the oxide region. The good correspondence between the simulated and experimental crystal morphologies (see Results and Discussion) justifies these simplifications a posteriori.

The second addendum in Eq. 1 accounts for the diffusion of $\mathrm{Ga}$ adatoms, with a mobility coefficient ${ }^{14,19}$ $M(\varphi) \sim \varphi^{2}(1-\varphi)^{2}$ in order to restrict the motion only at GaAs crystal surface. For the sake of simplicity, the same mobility is assumed for all facets. The driving force for material transfer is provided by the local gradient of the adatom chemical potential $\mu$, including both an energetic contribution at equilibrium, $\mu_{e q}$, and a kinetic term:

$$
\mu=\mu_{e q}+\varepsilon \tau(\hat{\mathbf{n}}) \frac{\partial \varphi}{\partial t}
$$

$\mu_{e q}$ is the functional derivative of the surface energy ${ }^{20}$, which is proportional to the profile curvature since the surface anisotropy is here neglected, as it plays a minor role in our kinetic regime, as discussed in the Supplementary Material. The kinetic term in Eq. (2) accounts for the incorporation dynamics ${ }^{13,21}$ with a kinetic coefficient $\tau(\hat{\mathbf{n}})$ that is dependent on the local faceting ${ }^{15,22}$. The physical meaning of this term can be clarified by reordering Eq. (2) as $\partial \varphi / \partial t \sim\left(\mu-\mu_{e q}\right) / \tau(\hat{\mathbf{n}})$. This shows that the progression of the growth front results directly from the difference between the local chemical potential of the adatoms $\mu$ and the one of the crystalline surface at equilibrium with the gaseous phase $\mu_{e q}$. In this respect, $\tau$ plays the role of adatom lifetime prior to the incorporation, and in general it depends on the availability and reactivity of both $\mathrm{Ga}$ and $\mathrm{As}$ at a given location. Under kinetic conditions, for convex morphologies, extended facets come from slower growing surfaces ${ }^{23,24}$, i.e. those with slower incorporation dynamics and longer adatom lifetime $\tau$. A continuum function $\tau(\hat{\mathbf{n}})$ is then devised similarly to Ref. 25, with maxima at all orientations of the crystal facets recognized experimentally, i.e. $\{111\} \mathrm{B}$, $\{110\}$ and $\{113\}$.

The time evolution, by coupling Eqs. (1) and (2), is numerically solved by Finite Element Method (FEM), using the AMDiS ${ }^{26,27}$ toolbox. Adaptive mesh refinement (see Fig. 2(c)) and a semi-implicit time-integration scheme are exploited to ensure high numerical accuracy and limit the computational load. Realistic length and time scales are considered in the simulations, so that the kinetic parameters are estimated by a best-morphology-fit procedure (see Supplementary Material for further details).

\section{RESULTS AND DISCUSSION}

The growth of GaAs fins is investigated by a close comparison between experimental data and numerical simulations based on the PF model illustrated in Sect. III. The analysis is structured in two stages. In the first one, we focus on the simplest case of vertical NMs in the $<11$ $2>$ direction, to assess the kinetic origin of the growth process. A close comparison between the samples at different growth stages and the results of the simulations for the morphological evolution enables us to setup the model and estimate the kinetic parameters for the two main facets $\{110\}$ and $\{111\} B$. Then, in the second stage, the variety of fin morphologies resulting by continuously changing the slit orientation along a circular pattern is explored. The adaptation of the crystal morphology to the slit alignment is investigated by both experimental SEM views and simulations. This will ultimately provide validation of the kinetic parameters, including also the one for the $\{113\}$ facets, demonstrating the reliability of the kinetic growth model.

\section{A. Kinetic growth of $<11-2>$-oriented vertical nanomembranes}

The kinetic origin of the NM growth process can be established by comparing samples after different deposition times. To this purpose, we find it convenient to analyze quantitatively the reference case of $<11-2>$-aligned vertical NMs. In particular, Fig. 3(a) reports SEM perspective views of NMs grown by 30,45 and $60 \mathrm{~min}$ long deposition. To appreciate the height variation, lateral profiles for these three stages are traced, as superimposed in Fig. 3(b). Evidently, the NM morphology evolves by extending the $\{110\}$ facets, reducing in turn the upper (111)B top. The narrow stripes of $\{113\}$ planes, as experimentally visible at the sides of the growth front, are not believed to play any active role in the evolution. To assess the most essential dynamics resulting in the growth of vertical NMs, we then first focus on the main $\{110\}$ and (111)B facets only, considering that $\{113\}$ just provide the connection for material transfer between them.

As shown in panel (b), almost the whole amount of incoming material contributes to the vertical growth, while the fin length and width change by just a few tens of nanometers. More quantitatively, systematic measurements from both top and perspective SEM views of the NM height vs. width variation in time returns a ratio in the growth velocities $v_{111 B} / v_{110}$ of at least 50 . Such a large difference is a key issue to provide the prominently vertical growth of the NM. This can be clarified further by exploiting a simple faceted growth model. According to Fig. 3(c), indeed, the shape evolution of a given NM profile can be just drawn geometrically by translating each facet plane according to its own velocity, as in the common Borgstrom construction method ${ }^{23}$. A full evolution sequence, starting from the experimental pro- 
file at 30 min growth, is reported in Fig. 3(d). A shape transition from trapezoidal to triangular is observed, as a consequence of the progressive extension of the slowest growing $\{110\}$ facets that consumes the fast (111)B growth front, until it disappears from the NM profile. Noticeably, the simulated shapes at 45 and 60 min nicely match the experimental ones.

The efficacy of this geometrical construction in capturing the experimental behavior makes clear the key role of the growth rates for different facets in controlling the NM morphology. However, it does not offer any insight on the growth mechanism. Although faster growth of the $\{111\}$ B facet is often observed in GaAs epitaxy ${ }^{2,28}$ (noticeably, it is commonly observed to result in nanowire growth), the large difference observed here with respect to the other $\{110\}$ facets deserves a careful explanation. Thermodynamic arguments can hardly provide an exhaustive motivation, as the typical variability of GaAs surface energies is on the order of a few tens of percent, according to literature studies ${ }^{11,29}$. If the deposited material had to move according only to the tendency toward surface energy minimization ${ }^{25}$, after filling the slit, it would possibly redistribute in a more isotropic shape by lateral overgrowth, similarly to the equilibrium Wulff shape (see Supplementary Material). The directionality of the MBE deposition flux is expected to favor vertical growth but, to fully account for the magnitude of the growth anisotropy observed in the experiments, significant differences in the material incorporation dynamics on the different facets must be expected, meaning that the NM growth is kinetically controlled.

The PF approach, defined in Sect. III, is well suited to simulate the morphological evolution of any fin during the growth, as resulting from the competition of deposition, diffusion and incorporation dynamics at the surface. Importantly, the faceting occurs spontaneously, as a consequence of the anisotropic properties of the material, without any a-priori assignment of the growth velocities and of the initial shape, as needed for the simple geometric approach. In a kinetic growth regime, the key parameter to be set in the model is the adatom lifetime before incorporation $\tau$, depending on the different crystal facet. As $\{110\}$ and $\{111\} \mathrm{B}$ facets are the main ones appearing in the NM shape, in a first approximation these are assumed to be the only relative maxima of $\tau$. According to the previous analysis, $\{110\}$ facets grow at the slowest rate, so that the corresponding incorporation time $\tau_{110}$ must be larger than the one for $\{111\} \mathrm{B}$ facets $\tau_{111 B}$. This looks reasonable by considering that the not-polar $\{110\}$ surface should be very flat at the atomic scale, thus making it hard to find stable incorporation sites. On the contrary, the $\{111\} \mathrm{B}$ polar surface probably exhibits not-stoichiometric reconstructions with more pronounced irregularities, favored by the out-of-equilibrium growth conditions, thus offering several sites for adatom incorporation. Additionally, literature studies ${ }^{2}$ suggest slow As incorporation on $\{110\}$ surfaces, resulting in a lower growth rate compared to $\{111\} \mathrm{B}$, at least for con- ditions leading to nanowire growth in MOCVD. A value of $\tau_{110}=10 \tau_{111 B}$ is estimated by means of a best-fit procedure between the simulated and the experimental morphologies, with $\tau_{111 B}=1$, for the adopted scaling of time. For the sake of simplicity, no anisotropy in surface energy is considered, i.e. all orientations are assumed to be energetically equivalent. As demonstrated in the Supplementary Material, this does not impact the simulation results, as the small differences in GaAs surface energies are ruled out by the larger differences in the kinetic parameters.

$\mathrm{PF}$ simulations are first applied to the reference case of the NM grown in $<11-2>$-oriented slits, as in Fig. 1 and 3. A full evolution sequence for the NM growth predicted by the PF kinetic model is shown in Fig. 4(a). The initial profile is set as a thin parallelepiped with the base matching the oxide slit size $1.5 \times 0.1 \mu \mathrm{m}^{2}$ and a small height of $0.01 \mu \mathrm{m}$, mimicking the pristine, almost conformal filling of the slit (considered as starting point in the simulations). We see that a spontaneous faceting, defined by all available $\{110\}$ facets and by the (111)B facet on the top, is promptly induced since the very first growth stages. Due to the elongated slit geometry, a trapezoidal shape is obtained. At first, the upper (111)B has the largest extension but, as deposition proceeds, the slanted $\{110\}$ planes tend to prevail causing a progressive shrinkage of the top. Finally, a triangular shape bounded by only the slowest $\{110\}$ facets is obtained. This matches well with the experimental observations and the geometrical analysis of Fig. 3.

In order to better understand the mechanism responsible for this strongly anisotropic growth, in Fig. 4(b) the chemical potential at the NM surface, including the kinetic term, is reported by a color map. During the growth process, $\mu$ is minimum at the (111)B top facet, i.e. where the incorporation dynamics is faster, so that material eventually diffuses away from the $\{110\}$ facets toward the top, as indicated by the arrows. A similar behavior was already reported for NW growth in SAE by MOCVD ${ }^{30,31}$. The difference in growth rate for the two facets inherently depends on the material transfer from one facet to the other and is mediated by the surface diffusion. As desorption is assumed to be negligible at the present MBE growth temperature of $625-635^{\circ} \mathrm{C}^{32}$, the whole amount of deposited material is subject to such a redistribution process, causing the facet growth rates to not be constant during the shape evolution. In particular, as the NM grows, the area of the $\{110\}$ facets increases with respect to the area of the (111)B facet, so that a larger amount of material is deposited on the $\{110\}$ regions and is then transferred to the shrinking NM top, causing an exponential increase in the vertical growth rate. This trend is reported in Fig. 4(c), where the NM height resulting from a growth simulation is plotted as a function of the deposition time. Here, an initial profile reproducing the experimental one after $30 \mathrm{~min}$ growth, displayed in Fig. 3, was set in order to directly compare the predictions of the model with the experi- 
(a)

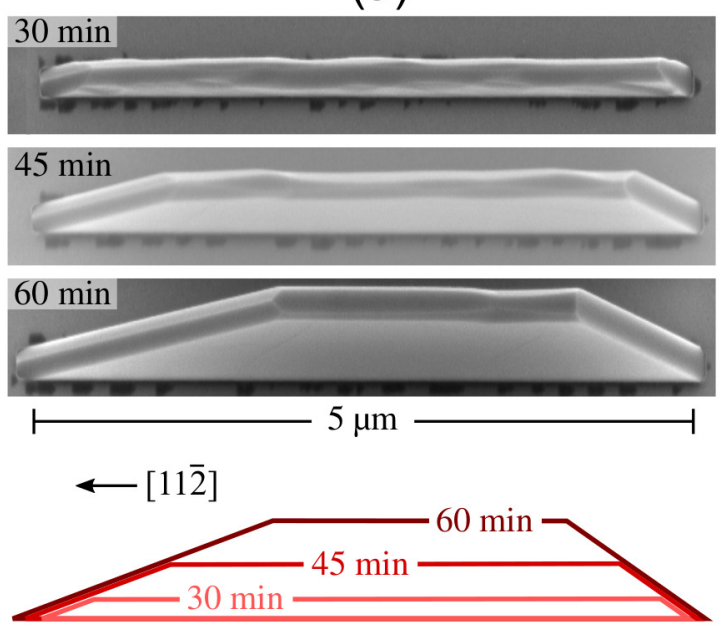

(b)

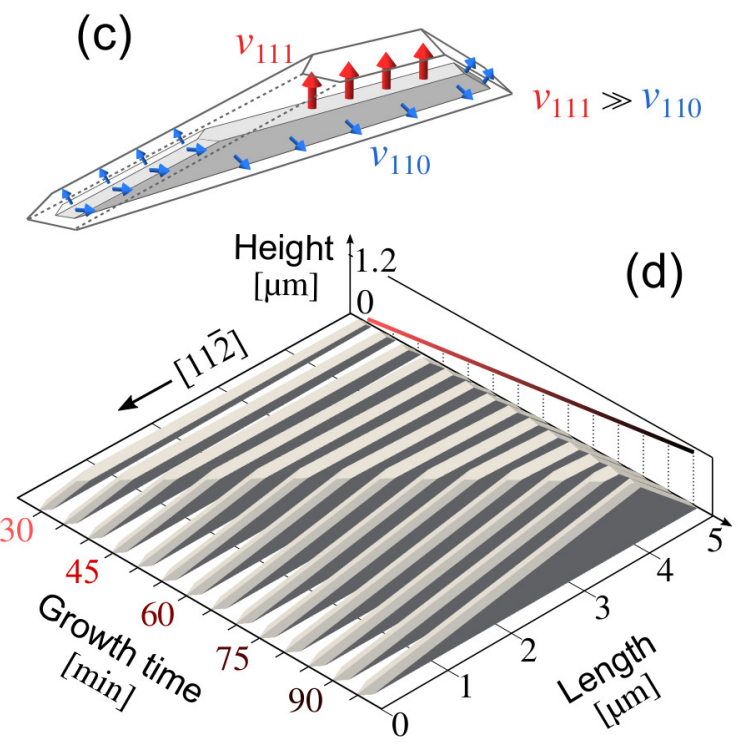

FIG. 3. Assessing the facet growth rates in $<11-2>$-oriented vertical NMs. (a) SEM perspective views of a single NM grown after 30, 45 and 60 min of GaAs deposition. (b) Lateral profiles drawn for the three stages of panel (a). (c) Schematics of the facet growth by assigning different velocities $v$ for the $\{110\}$ and (111)B facets forming the NM. The velocity of the (111)B, $v_{111 B}$, is deduced to be much larger than the one of $\{110\}$ facets, $v_{110}$, according to the experimental findings. (d) Growth sequence geometrically reconstructed according to the procedure in panel (c), starting from a profile matching the one at 30 min in panel (a).

mental growth sequence, as indicated by the dots. This one-to-one comparison also allows us to attribute a realistic time unit to the otherwise arbitrary timescale of simulations.

We find it very instructive to inspect by simulations the growth of NM developing from slits of different length (from $0.5 \mu \mathrm{m}$ to $5 \mu \mathrm{m}$ ), still with the same width of $100 \mathrm{~nm}$. A complete analysis is reported in Fig. 5, showing the variation of the NM height as a function of both the deposition time (panel (a) ) and the slit length $L$ (b), along with top views of the NM profiles after the same simulation time of $22 \mathrm{~min}$. In all cases, the (111)B top facet shrinks as the $\{110\}$ facets tend to enclose the shape toward a $\{110\}$-only triangular shape: obviously, the shorter the slit is, the sooner the facet at the top disappears. The onset of this transition is clearly recognizable in the abrupt change in the slope of the curves for 0.5 and $1 \mu \mathrm{m}$ long slits in panel (a). Indeed, as soon as the fast-growing (111)B top disappears, the deposited material accumulates on all the exposed $\{110\}$ facets and incorporates there at a slower rate. As the effect of the different incidence angles of the MBE beam on each $\{110\}$ facet is minor, a more self-similar growth occurs, characterized by lateral expansion comparable to the vertical one, as clearly seen in the shortest simulation profiles of Fig. 5(c). In the limit of a square mask opening, a perfectly 3 -fold symmetric pyramidal dot is formed, bounded by the three $\{110\}$ facets, forming the back and front of the elongated NMs.

Furthermore, by comparing the different curves of Fig. 5(a), it is notable how the vertical growth follows a slightly different exponential curve, depending on the slit length, slower for the most elongated case. Correspondingly, at fixed deposition time, the NMs grown from shorter slits are found to be taller than the most elongated ones as shown in panel (b). Therein, simulation results (red curve) are compared to experimental data (grey band), collected from multiple samples after 30 min deposition, showing a tendency consistent with the theoretical prediction. Following the concept of the kinetic model, this is straightforwardly explained by noting that, for shorter structures the material deposited on the $\{110\}$ facets spreads over a (111)B facet of smaller area.

\section{B. Three-dimensional fin growth on a circular pattern}

The study of fin growth while changing the slit orientation (with respect to the $<11-2>$ direction considered in the previous section) offers a deeper insight into the kinetic mechanism controlling the crystal formation and faceting. Moreover, it makes it possible to fine-tune the kinetic model, by testing the validity of the estimated incorporation times. A circular pattern for testing the angular dependence of growth was designed for this purpose. It consisted of slits etched in a ring pattern with an angular resolution $<1^{\circ}$. By depositing GaAs under the same conditions considered for $<11-2>$-oriented patterns, 3D faceted fins are observed for intermediate orientations, as illustrated in Fig. 6(a). A closer inspection 

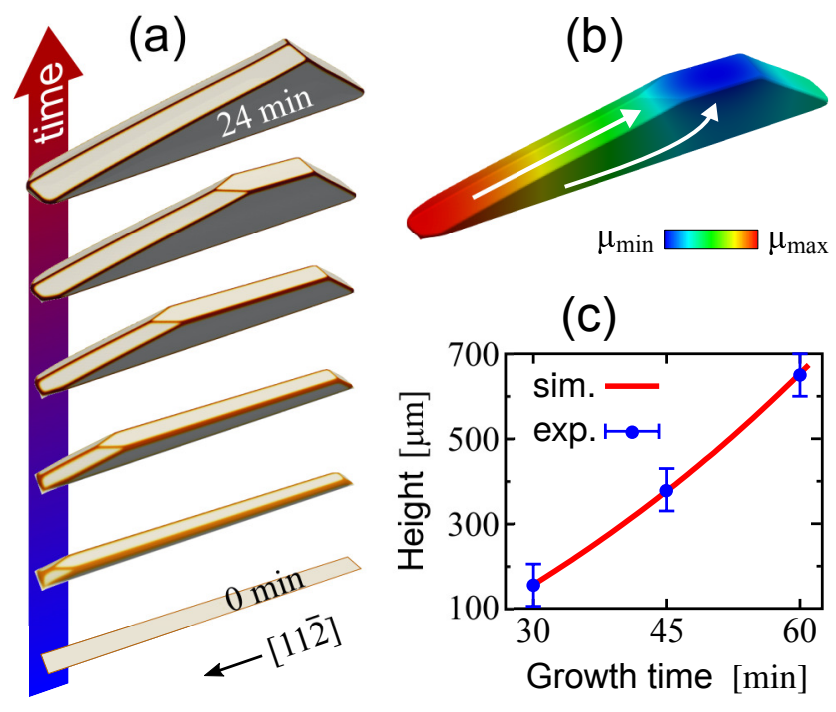

FIG. 4. PF simulation results for vertical NMs. (a) Growth sequence of a $\mathrm{NM}$ in a $1.5 \mu \mathrm{m}$ slit oriented along [11-2]. The deposited material tends to diffuse from the lateral $\{110\}$ facets to the top (111)B, according to the gradient of the chemical potential $\mu$, which is represented in the color map in panel (b). The white arrows sketch the flow of material on the surface. (c) Comparison of the NMs height between simulation (red line) and experimental data points, retrieved by SEM images, in the case of a $5 \mu \mathrm{m}$ slit. The error bar represents the uncertainty in the measurements.

(panel (b)) reveals an almost continuous change in morphology, from vertical to slanted NMs. Enlarged views of these two limiting morphologies are shown in panels (c) and (d) respectively.

The vertical NMs develop from $<11-2>$-oriented slits as widely discussed in the previous section with the same $\{110\}$-faceted trapezoidal shape. The slanted ones form along $<110>$ directions and are shaped as isosceles trapezoids with a highly defected top. As made evident in Fig. 6(e), their sidewalls form an angle of $\sim 35^{\circ}$ with the substrate plane which indicates them to be composed of slow-growing $\{110\}$ facets. Indeed, $<11-2>$ and $<110>$ directions are the only ones on the (111)B substrate plane to admit a pair of $\{110\}$ facets running along the slit length, forming the fin sidewalls. Furthermore, a careful analysis of the slanted morphology allows us to conclude that all exposed facets belong to the $\{110\}$ family, except for the irregular, indented growth front at the top, possibly composed of $\{11 \mathrm{n}\}$ facets. This is consistent with the kinetic growth mechanism depicted in the previous section, i.e. the growing shape is dominated by the slowest $\{110\}$ facets available while the fast-advancing growth front is provided by $\{111\}$ and $\{11 n\}$ facets. The simple geometrical analysis illustrated in Fig. 3(c) can also be applied to predict the evolution of these $<110>$-oriented structures, as done in Fig. 6(f). The initial morphology is set in such a way to match the experimental shape of Fig. 6(d) at $60 \mathrm{~min}$ deposition and its evolution is de- (a)
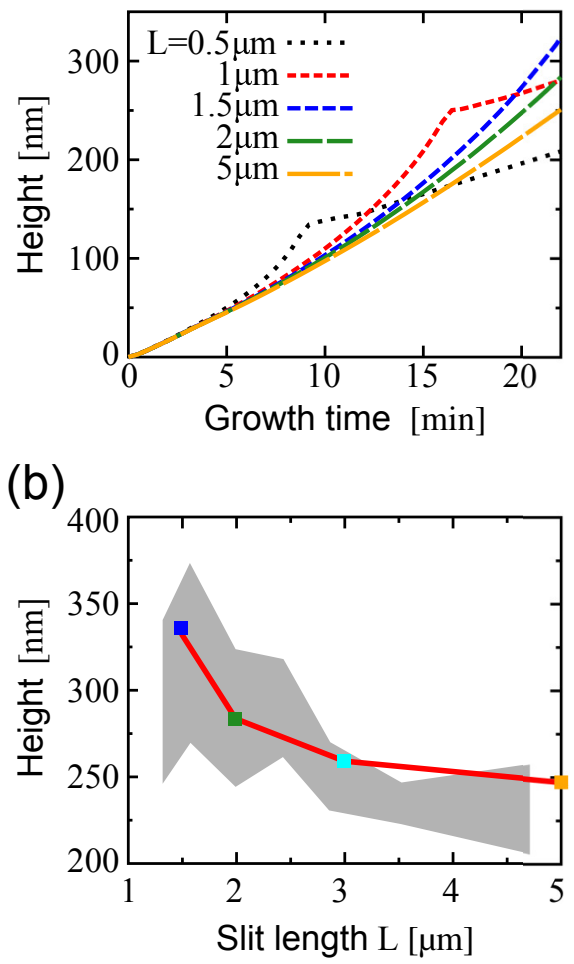

(c)

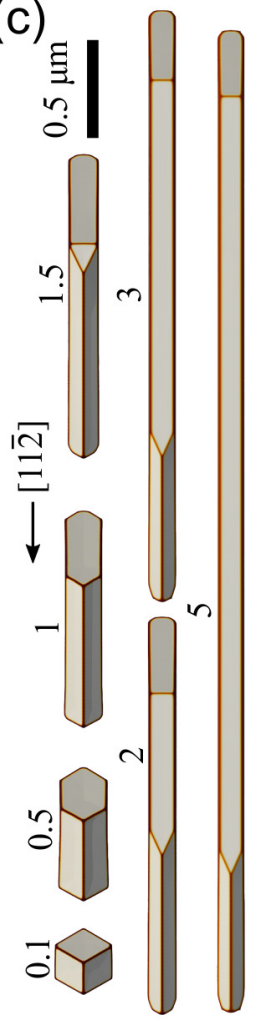

FIG. 5. Comparison of the nanomembranes height for different lengths of the oxide slit. (a) Evolution in time of the NM height. The sudden variation in the slope observed for the cases of 0.5 and $1 \mu \mathrm{m}$ corresponds to the disappearance of the top (111)B facet. (b) Comparison between the fin height predicted by simulations and the corresponding range of heights measured experimentally after $30 \mathrm{~min}$ deposition (grey band). (c) Top view of the simulated profiles (at $22 \mathrm{~min}$ ) for the different labelled lengths.

termined by using the very same velocity parameters extrapolated for the vertical NM of Fig. 3(d). For the sake of simplicity, the growth front is just set by two straight $\{111\} \mathrm{B}$ facets. It is predicted to gradually shrink and finally disappear resulting in a slanted triangular shape.

Due to the lattice symmetry, on the (111)B substrate plane there are six $<11-2>$ available directions alternated with six $<110\rangle$ directions, at $30^{\circ}$ one from the other. Even if the same fin morphologies reflect this repetition, the actual rotational symmetry is only three-fold. Indeed, the fin back and front facets exchange from the inner to the outer side of the circle when moving by $30^{\circ}$ from one $\langle 11-2\rangle$ direction to the next one (e.g. [2-1$1]$ and $[-12-1]$ ), as made evident in Fig. $6(\mathrm{~g})$, where the $\{110\}$ facet forming the back of the fin shape is highlighted in yellow. This occurs gradually trough all intermediate fin shapes and the actual reversal happens when reaching the $\langle 110\rangle$ direction in between, where front and back facets are equivalent. Analogously, the side to which the slanted NMs are tilted alternates from right to left in adjacent $<110>$ directions (e.g. [-110] and [01- 

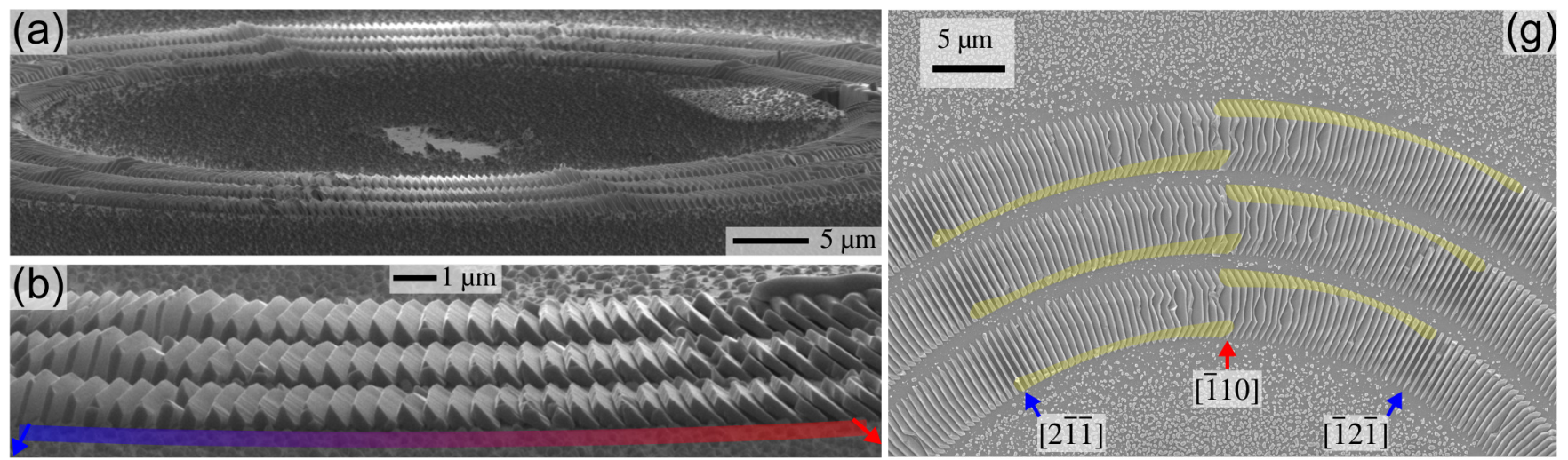
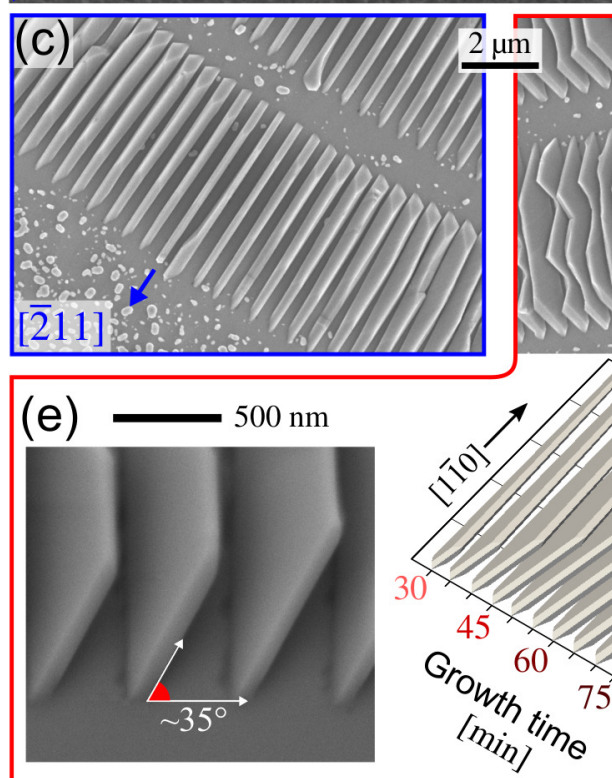

90

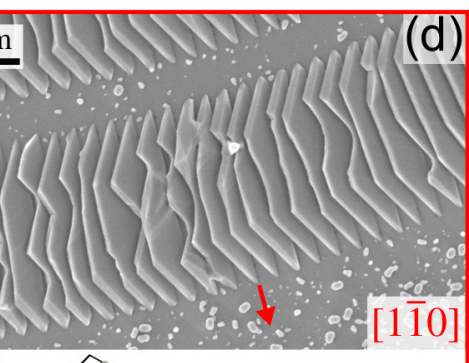

(d)

\section{(1)}

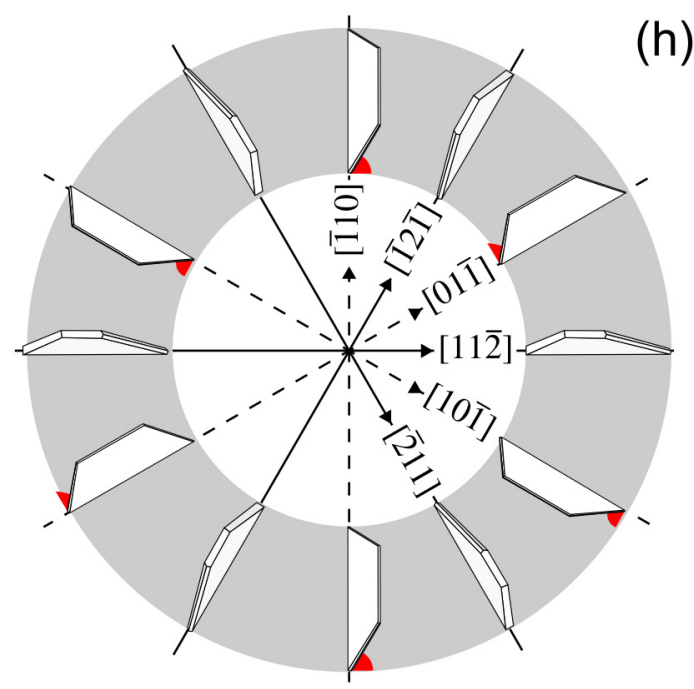

(f)

FIG. 6. Fin growth on a circular pattern obtained by rotating the slit for every $<1^{\circ}$ after 60 min GaAs deposition. (a) SEM view of the whole patterned area showing selective growth into all slits. (b) Enlarged view of a $30^{\circ}$ wide sector of panel (a) showing the continuous change in the NM morphology from vertical to slanted shapes. Top views of these two limiting cases are shown in panels (c) and (d) respectively. The actual inclination of the slanted NMs is made evident in panel (e), showing an SEM image taken from the fin front. (f) Growth sequence reproduced by the geometric construction of Fig. 3(c). The same growth velocities $v_{111 B}$ and $v_{110}$ of Fig. $3(\mathrm{~d})$ are considered, and the morphology is seen to match that of the experiment after 60 min deposition. (g) SEM top view of a $30^{\circ}$ sector in between successive $<11-2>$ directions showing the exchange from inner to outer of the 110 back facet of the fin, colored in yellow. (h) Scheme of the three-fold repetition of vertical and slanted morphologies along the whole circle.

1]). The schematics in Fig. 6(h) shows the full symmetry along the circular pattern for the two principal shapes.

PF simulations can be straightforwardly applied to simulate the fin growth on arbitrarily oriented slits, by simply rotating the initial profile in the reference frame. The outcome of growth simulations performed for slit orientations varying from [11-2] to [01-1] directions is illustrated in Fig. 7. In order to obtain an accurate representation of the morphology, $\{113\}$ facets were also included in the simulations as another set of relative maxima in the incorporation time function. Their presence was recognized experimentally at the top of the vertical NMs and it is likely to occur in the other slit orientations, where $\{11 \mathrm{n}\}$ segments are found. Indeed, $\{113\}$ planes are singular and their surface energy is particularly low, comparable to the low-index facets ${ }^{29}$, making them good candidates to appear in the crystal shape (actually, $\{114\}$ facets could also appear due to their low energy ${ }^{33}$ while, on the contrary, $\{112\}$ facets are unstable ${ }^{29}$ ). By searching for the best fit of the experimental morphologies, the adatom lifetime on $\{113\}$ facets was estimated to be twice the value of the one on $\{111\} \mathrm{B}\left(\tau_{113}=2 \tau_{111 B}\right)$, thus returning an intermediate growth rate between the fastest $\{111\} \mathrm{B}$ front and the slow $\{110\}$ facets. This could be justified by considering that $\{113\}$ facets are not as flat as $\{110\}$ surfaces, therefore providing intermediate incorporation sites (for example: step edges).

A progressive transition in the shape, from vertical to 


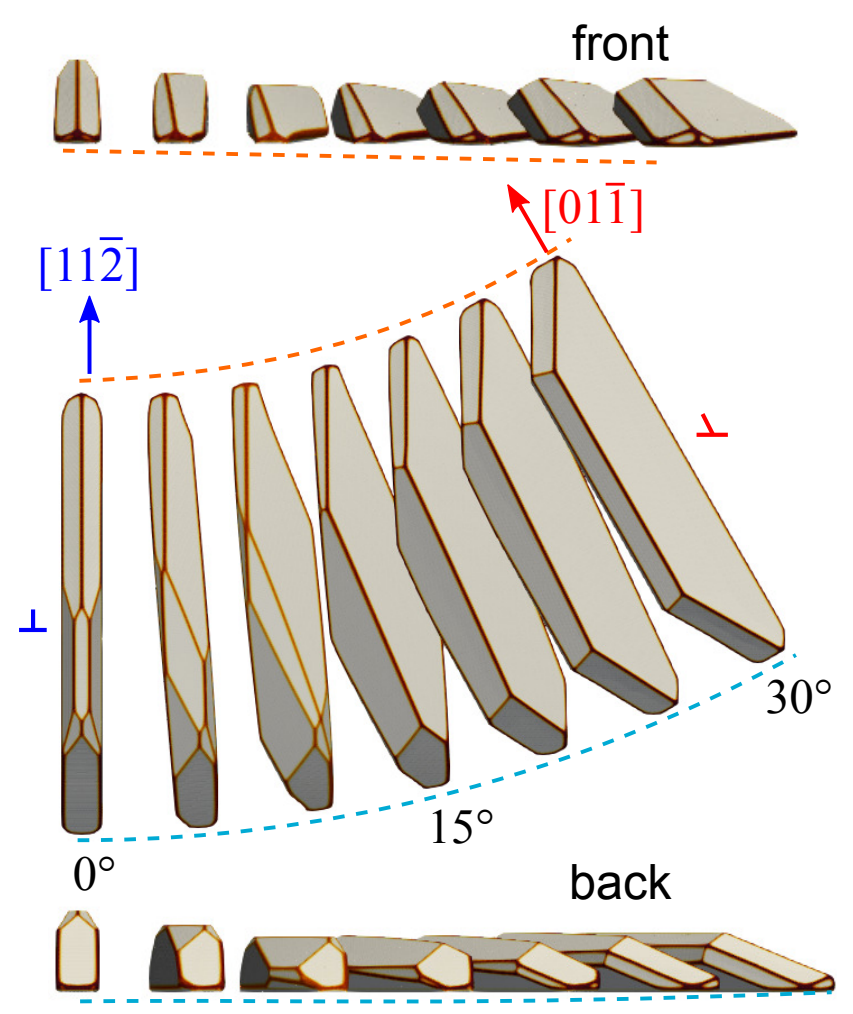

FIG. 7. Fin profiles predicted by growth simulations for different orientations of a $1.5 \mu \mathrm{m}$ slit. The rotation angles for the slit range from $0^{\circ}$ to $30^{\circ}$, with $5^{\circ}$ step. Vertical and slanted NMs are found at $0^{\circ}$ and $30^{\circ}$ respectively, while multifaceted fins are obtained for intermediate orientations. As evident in the front and back views, the fin height is maximum for the $0^{\circ}$-oriented $\mathrm{NMs}$ and decreases to a minimum at $15^{\circ}$ and slightly increases again while moving toward $30^{\circ} .\{110\}$, $\{111\} \mathrm{B}$ and $\{113\}$ facets are considered in the simulation.

slanted, is evident when rotating the slit by $30^{\circ}$, closely resembling the experimental view in Fig. 6(b). Each morphology results by a different composition of the same set of the available $\{110\},\{111\} B$ and $\{113\}$ facets with traits resembling more the vertical or slanted shape according to the closest orientation. For small misorientation angles from $<11-2>$ or $<01-1>$ direction, the growing fin tends to slightly rotate to re-align along the favorable axis. A substantial asymmetry is evident for larger misalignments, at around $15^{\circ}$.

The morphologies of the simulated fins are detailed in Fig. 8. Panel (a) shows the vertical NM, grown from the [11-2]-oriented slit. The profile is similar to the one shown in Fig. 4, except for the presence of $\{113\}$ facets running side-by-side to the upper (111)B facet, in better agreement with experiment. A slanted NM formed along the [01-1] direction is instead shown in panel (b). As in the experiments (Fig. 6(d)), the simulated growth process makes it more convenient to develop an inclined shape bounded by $\{110\}$ facets rather than spread laterally on the oxide. The growth front at the crystal top consists of just two $\{113\}$ facets, in place of the $\{111\} \mathrm{B}$ facets considered in the geometrical reconstruction in Fig. 6(f). This is a direct consequence of the choice of a longer incorporation time $\tau_{113}$ with respect to the one on $\{111\} \mathrm{B}$, resulting in a slower growth rate for the $\{113\}$ facets, that makes them prevail, after a short coexistence with $\{111\} \mathrm{B}$ at the very early stages. Consequently, the slanted NMs grow slightly thicker while reaching a lower height compared to the vertical one, as observed experimentally.

The faceted fin geometry resulting by simulations for slits misaligned by $15^{\circ}$ with respect to a $<11-2>$ (or $<110>$ ) direction is reported in Fig. 8(c). In this case, the facets tend to align both along $<11-2>$ and $<110>$ directions (see top view). The resulting shape thus contains $\{110\}$ facets present in both the vertical NM of Fig. 8(a), e.g. those labeled as 4 and 6 , and in the slanted one of Fig. 8(b), e.g. those labeled as 1 and 5. Consequently, as shown by the cross sections in Fig. 8(c), the lateral growth of the fin is larger, extending above the oxide well beyond the slit (shown by dotted lines). This morphology looks consistent with the experimental data, in particular with the magnifications of the corresponding portion of the circular pattern reported in Fig. 8(d), even if the facets are less defined. Both simulation and experiments agree on the existence of an edge at the top of the fin, possibly formed by the connection of a $\{110\}$ slanted facet (labeled as 1) and a $\{113\}$ facet (labeled as $\alpha)$.

Irregularities in the experimental profiles are mostly observed at the crystal top and they could relate to growth imperfections, as seen for the slanted NMs. Interestingly, the gray-scale contrast of the SEM images reveals striping all along the elongated facets, which suggests a high density of steps, probably formed to facilitate a rotation of the crystal morphology towards the actual $\{110\}$ and $\{113\}$ orientations. These atomic-scale features (not included in our model) can be directly associated to the existence of a small curvature within the facets appearing in the simulation profiles. For example, the inclination of the largest $\{110\}$ facet in Fig. 8(c) is observed to vary of about $3^{\circ}$ along its whole length. This is a direct consequence of the constraint imposed by the slit orientation. Indeed, during the first stages, material is forced to accumulate along that unfavorable direction, but as soon as overgrowth on the oxide region becomes possible, a rearrangement in the shape occurs spontaneously.

It is worth noting how, for the misaligned configurations, $\{113\}$ facets, negligible in the vertical NMs, compete more directly in the material redistribution, developing over relatively large areas. Indeed, steps and step bunching are expected to increase the growth velocity of $\{110\}$ facets, reducing the imbalance with the (111)B and $\{113\}$ ones, so that the latter have both a geometric and kinetic role in closing the surface profile, when the solid is no longer a nearly two-dimensional one. 


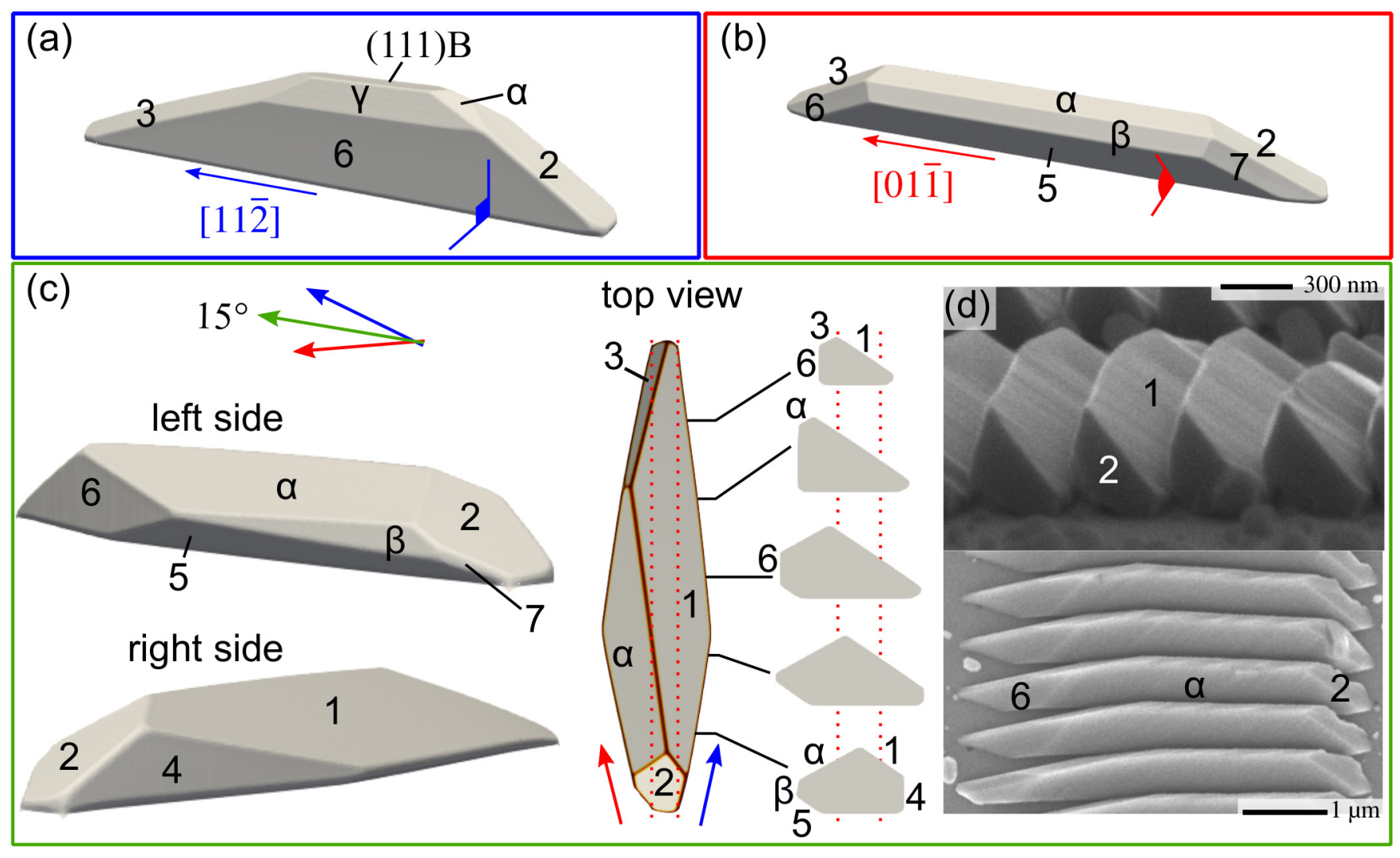

$1-6:\{110\} \quad \alpha, \beta, \gamma:\{113\}$

FIG. 8. Details of the simulated fin morphologies reported in Fig. 7 for three slit orientations: (a) vertical NM at $0^{\circ}$; (b) slanted $\mathrm{NM}$ at $30^{\circ}$; (c) multi-faceted fin structure at $15^{\circ}$, with coexistence of facets from both the $0^{\circ}$-oriented NM shape (facets 4 and 6 ) and the $30^{\circ}$ one (facets 1 and 5). Different vertical cross-sections of the $15^{\circ}$ fin are also reported, aligned with respect to the oxide slit, traced by the dotted lines, and show a large lateral overgrowth reflecting the tendency to realign with the $0^{\circ}$ and $30^{\circ}$ NMs. (d) SEM images corresponding to the $15^{\circ}$ orientation, resembling the simulation results in panel (c). Steps and irregularities are distinguishable on the fin surfaces.

\section{CONCLUSIONS}

The kinetic growth origin of GaAs homoepitaxial NMs by SAE-MBE has been assessed, showing that the morphology changes from 2D NMs to $3 \mathrm{D}$ faceted fins, depending on the slit orientation. These results, quite useful for novel applications, allow to extract more fundamental information on the kinetic growth velocities of the GaAs facets, responsible for the morphology of different nanostructures. In order to identify the kinetic growth parameters for the different facets, fins were grown in a circular array of slits, causing different facets to appear. Their morphologies were then interpreted by a phasefield kinetic-growth model. Despite the limitations inherent in the continuum treatment of the crystal structure, omitting any atomic-scale detail such as the steps and step-bunching recognized by SEM on $\{110\}$ facets in misoriented fins, this approach proved to be very effective in capturing the physics behind the growth of such largescale structures, not accessible by methods with atomistic resolution. The simulation results, in particular those in- cluding the full set of $\{111\} B,\{110\}$ and $\{113\}$ facets, look consistent with the experimental observation of the fin morphologies for any slit orientation.

The crystal-incorporation times fitted to the experimental SEM images reveal an interesting hierarchy in the facet growth rates: $\{111\} \mathrm{B}>\{113\} \gg\{110\}$. This could not be simply inferred on the basis of their relative surface energies, i.e. high surface energy corresponds to high growth rate, a popular concept lacking quantitative value. In fact, it misses the complex mechanisms of adatom redistribution and incorporation at the surface, which appears to make a larger difference in growth rates than the surface energies themselves. Well-oriented NMs are a clear example of this, as $\{111\} \mathrm{B}$ facets are found to grow much faster, $\sim 50$ times, than $\{110\}$ facets, despite having comparable surface energies, $\{111\} \mathrm{B}$ facets being even slightly more stable than $\{110\}$ facets. 


\section{ACKNOWLEDGMENTS}

We thank Prof. Stefano Sanguinetti (University of Milano-Bicocca) for fruitful discussion. We acknowledge financial support from National Science Foundation through projects No. IZLRZ2_163861 and 200021_169908 and the NCCR QSIT and from the Eu- ropean Union through Horizon-2020 FET " $\mu$-SPIRE" project, id 766955. M.S acknowledges the support of the Postdoctoral Research Fellowship awarded by the Alexander von Humboldt Foundation. We acknowledge computational resources from CINECA (Italy), Center for Information Services and High Performance Computing (ZIH) at TU-Dresden, and Jülich Supercomputing Center within the Project No. HDR06.
* Theory: roberto.bergamaschini@unimib.it

† Experiments: martin.friedl@epfl.ch

¥ Present address: The Filler Laboratory, Chemical and Biomolecular Engineering, Georgia Institute of Technology, Atlanta, GA, USA

1 K. Tomioka, Y. Kobayashi, J. Motohisa, S. Hara, and T. Fukui, Nanotechnology 20, 145302 (2009).

${ }^{2}$ K. Tomioka, K. Ikejiri, T. Tanaka, J. Motohisa, S. Hara, K. Hiruma, and T. Fukui, J. Mater. Res. 26, 2127 (2011).

${ }^{3}$ K. P. Bassett, P. K. Mohseni, and X. Li, Appl. Phys. Lett. 106, 133102 (2015).

4 C.-Y. Chi, C.-C. Chang, S. Hu, T.-W. Yeh, S. B. Cronin, and P. D. Dapkus, Nano Lett. 13, 2506 (2013).

5 S. Arab, C.-Y. Chi, T. Shi, Y. Wang, D. P. Dapkus, H. E. Jackson, L. M. Smith, and S. B. Cronin, ACS Nano 9, 1336 (2015).

6 G. Tütüncüoglu, M. de la Mata, D. Deiana, H. Potts, F. Matteini, J. Arbiol, and A. Fontcuberta i Morral, Nanoscale 7, 19453 (2015).

7 Z. Yang, A. Surrente, G. Tütüncüoglu, K. Galkowski, M. Cazaban-Carrazé, F. Amaduzzi, P. Leroux, D. K. Maude, A. Fontcuberta i Morral, and P. Plochocka, Nano Lett. 17, 2979 (2017).

8 H. Potts, Y. van Hees, G. Tütüncüoglu, M. Friedl, J.-B. Leran, and A. Fontcuberta i Morral, Cryst. Growth Des. 17, 3596 (2017).

9 M. Friedl, K. Cerveny, P. Weigele, G. Tütüncüoglu, S. Martí-Sánchez, C. Huang, T. Patlatiuk, H. Potts, Z. Sun, M. O. Hill, L. Güniat, W. Kim, M. Zamani, V. G. Dubrovskii, J. Arbiol, L. J. Lauhon, D. M. Zumbühl, and A. Fontcuberta i Morral, Nano Lett. 18, 2666 (2018).

10 F. Krizek, J. E. Sestoft, P. Aseev, S. Marti-Sanchez, S. Vaitiekenas, L. Casparis, S. A. Khan, Y. Liu, T. Stankevic, A. M. Whiticar, A. Fursina, F. Boekhout, R. Koops, E. Uccelli, L. P. Kouwenhoven, C. M. Marcus, J. Arbiol, and P. Krogstrup, arXiv:1802.07808.

11 N. Moll, A. Kley, E. Pehlke, and M. Scheffler, Phys. Rev. B 54, 8844 (1996).

12 J. Hartmann, F. Steib, H. Zhou, J. Ledig, S. Fündling, F. Albrecht, T. Schimpke, A. Avramescu, T. Varghese, H. H. Wehmann, M. Straßburg, H. J. Lugauer, and A. Waag, Cryst. Growth Des. 16, 1458 (2016).

13 J. W. Cahn and J. E. Taylor, Acta Metall. Mater. 42, 1045 (1994).
14 B. Li, J. Lowengrub, A. Rätz, and A. Voigt, Commun. Comput. Phys. 6, 433 (2009).

15 A. Rätz, A. Ribalta, and A. Voigt, J. Comput. Phys. 214, 187 (2006).

16 J. Tersoff, M. D. Johnson, and B. G. Orr, Phys. Rev. Lett. 78, 282 (1997).

17 R. Bergamaschini, S. Bietti, A. Castellano, C. Frigeri, C. V. Falub, A. Scaccabarozzi, M. Bollani, H. von Känel, L. Miglio, and S. Sanguinetti, J. Appl. Phys. 120, 245702 (2016).

18 M. Salvalaglio, R. Backofen, and A. Voigt, Phys. Rev. B 94, 235432 (2016).

19 A. Voigt, Appl. Phys. Lett. 108, 2015 (2016).

20 S. Torabi, J. Lowengrub, A. Voigt, and S. Wise, Proc. R. Soc. A 465, 1337 (2009).

21 E. Fried and M. E. Gurtin, Adv. Appl. Mech. 40, 1 (2004).

22 C. Stöcker and A. Voigt, J. Cryst. Growth 303, 90 (2007).

23 S. H. Jones, L. K. Siedel, and K. M. Lau, J. Cryst. Growth 108, 73 (1991).

24 J. W. Cahn and W. C. Carter, Metall. Mater. Trans. A 27A, 1431 (1996).

25 M. Salvalaglio, R. Backofen, R. Bergamaschini, F. Montalenti, and A. Voigt, Cryst. Growth Des. 15, 2787 (2015).

26 S. Vey and A. Voigt, Comput. Vis. Sci. 10, 57 (2007).

27 T. Witkowski, S. Ling, S. Praetorius, and A. Voigt, Adv. Comput. Math. 41, 1145 (2015).

28 M. Knoedler, N. Bologna, H. Schmid, M. Borg, K. E. Moselund, S. Wirths, M. D. Rossell, and H. Riel, Cryst. Growth Des. 17, 6297 (2017).

29 J. Platen, A. Kley, C. Setzer, K. Jacobi, P. Ruggerone, and M. Scheffler, J. Appl. Phys. 85, 3597 (1999).

30 J. Jin, T. Stoica, S. Trellenkamp, Y. Chen, N. Anttu, V. Migunov, R. M. Kawabata, P. J. Buenconsejo, Y. M. Lam, F. Haas, H. Hardtdegen, D. Grützmacher, and B. E. Kardynał, ACS Appl. Mater. Interfaces 8, 22484 (2016).

31 M. Yao, C. Sheng, M. Ge, C. Y. Chi, S. Cong, A. Nakano, P. D. Dapkus, and C. Zhou, ACS Nano 10, 2424 (2016).

${ }^{32}$ T. Kojima, N. J. Kawai, T. Nakagawa, K. Ohta, T. Sakamoto, and M. Kawashima, Appl. Phys. Lett. 47, 286 (1985).

33 J. Márquez, P. Kratzer, L. Geelhaar, K. Jacobi, and M. Scheffler, Phys. Rev. Lett. 86, 115 (2001). 